\title{
A note on order paracompactness
}

\section{M.K. Singal and Shashi Prabha Arya}

\begin{abstract}
This paper contains some results on order paracompact spaces. Some of the results offer improvements of some of the results of McCandless in Canad. J. Math. 21 (1969). Some other results of McCandless are deduced as corollaries from our results. The concepts of order closure preserving and order cushioned collections are introduced and using these, characterizations of paracompactness in regular spaces are obtained.
\end{abstract}

McCandless [4] has initiated a study of order paracompact spaces. This class of spaces contains the class of order totally-paracompact spaces defined by Fitzpatrick and Ford [2] which is an important class, since the class of order totally-paracompact metric spaces is the largest known class of spaces for which the large and small inductive dimensions coincide. The main result proved by McCandless is that every regular, order paracompact space is collectionwise normal. With the help of this result and a result of Tamano [7], he proves that a regular $T_{1}$-space is order paracompact if. and only if it is paracompact. We shall prove that this result holds even without the assumption of the space being $T_{1}$. From this will follow as a corollary the main result of McCandless mentioned above. McCandless has proved a result concerning direct preservation of order paracompactness under mappings. We shall show that order paracompactness is inversely preserved under mappings which are closed, continuous, with point inverses compact. As a corollary to this result follows another result of McCandless - namely, the product of an order paracompact space with a compact space is order paracompact. In the end we introduce some new concepts and obtain some new characterizations of paracompact spaces.

Received 13 November 1970. 
DEFINITION 1. A space $X$ is said to be order paracompact if every open covering $U$ of $X$ has an open refinement $V$ which is linearly ordered and is such that for each $V \in V$, the family $\left\{V^{\prime}: V^{\prime}<V, V^{\prime} \in V\right\}$ is locally finite at each point of $\bar{V}$.

DEFINITION 2. Call a space $X$ strongly order paracompact if every open covering of $X$ has a linearly locally finite open refinement, where by a linearly locally finite family is meant a linearly ordered family $u$ such that for each $U \in U,\left\{U^{\prime}: U^{\prime} \in U, U^{\prime}<U\right\}$ is locally finite.

DEFINITION 3. Call a space $X$ weakly order paracompact if every open covering of $X$ has an order locally finite open refinement, where by an order locally finite family is meant a family $U$ which is linearly ordered and is such that $\left\{U^{\prime}: U^{\prime}<U\right\}$ is locally finite at each point of $U$ for each $U \in U$.

The definition of order locally finite families is due to Katuta [3].

Obviously, strongly order paracompact implies order paracompact, and this implies weakly order paracompact.

It is easily seen that every $\sigma$-locally finite family is linearly locally finite. Therefore, a space every open covering of which has a $\sigma$-locally finite open refinement is strongly order paracompact. In particular, every Lindelöf space is strongly order paracompact.

It has been proved by Vaughan [8] that a regular space is paracompact iff it is strongly order paracompact. Also, Katuta has shown that in a regular space weak order paracompactness is equivalent to paracompactness. It therefore follows that a regular space is paracompact if and only if it is order paracompact. However, we give here a proof of this fact for the sake of completeness.

THEOREM 1. For a regular space $X$, the following are equivalent:

(a) $X$ is paracompact;

(b) $X$ is strongly order paracompact;

(c) $X$ is order paracompact;

(d) $X$ is weakly order paracompact.

Proof. $(a) \Rightarrow(b) \Rightarrow(c) \Rightarrow(d)$ is obvious. We prove that $(d) \Rightarrow(a)$. 
Let $u$ be any open covering of $X$. Then there exists an order locally finite open refinement $V$ of $u$. For each $V \in V$, let $W_{V .}=V \sim U\left\{V^{\prime}: V^{\prime}<V, V^{\prime} \in V\right\}$. Let $W=\left\{W_{V}: V \in V\right\}$. We shall prove that $W$ is a locally finite refinement of $U$. First, let $x \in X$. Then there exists $V \in V$ such that $x \in V$. Since $\left\{V^{\prime}: V^{\prime}<V\right\}$ is to be locally finite at each point of $V$, therefore $x$ can belong to at most finitely many members of $\left\{V^{\prime}: V^{\prime}<V\right\}$, say $x \in V_{i}: i=1,2, \ldots, n$ and $V_{1}<V_{2}<V_{3}<\ldots<V_{n}$. Then $x \notin V^{\prime}$ if $V^{\prime}<V_{1}$ and therefore $x \in W_{V_{1}}$. Thus $W$ is a covering of $X$ each member of which is contained in some member of $U$. It remains to be proved now that $w$ is locally finite. There exists an open set $M$ such that $x \in M$ and $M$ intersects finitely many members of $\left\{V^{\prime}: V^{\prime}<V\right\}$. Also, $V \cap V^{*}=\emptyset$ for all $V^{*} \in V$ such that $V^{*}>V$. Hence $M \cap V$ is a neighbourhood of $x$ which intersects finitely many members of $W$. Thus every open covering of $X$ has a locally finite refinement. Therefore $X$, being regular, is paracompact (cf. Michael [5]).

COROLLARY. (McCandless [4], Theorem 3). Every regular order paracompact space is collectionise normal.

Proof. Every regular, order paracompact space is paracompact and it is known that every paracompact, regular space is collectionwise normal (cf. Bing [1]).

THEOREM 2. If $f$ is a closed, continuous mapping of a space $X$ onto a space $Y$ such that $f^{-1}(y)$ is compact for each $y \in Y$, then $X$ is order paracompact if $Y$ is 80 .

Proof. Let $U=\left\{U_{\alpha}: \alpha \in \Lambda\right\}$ be any open covering of $X$. Let $F$ denote the family of all finite subsets $\gamma$ of $\Lambda$. Since $f^{-1}(y)$ is compact for each $y \in Y$, therefore for each $y \in Y$, there exists a finite subset $\gamma$ of $\Lambda$ such that $f^{-1}(y) \subseteq \underset{\alpha \in \gamma}{U} U_{\alpha}$. Let now $V_{\gamma}=Y \sim f\left(X \sim \underset{\alpha \in \gamma}{U} U_{\alpha}\right)$. Since $f$ is closed, therefore $V_{\gamma}$ is open. Also, $y \in V_{\gamma}$ and $f^{-1}\left(V_{\gamma}\right) \subseteq \underset{\alpha \in \gamma}{U} U_{\alpha}$. Now, $V=\left\{V_{\gamma}: \gamma \in F\right\}$ is an open 
covering of $Y$. Since $Y$ is order paracompact, there exists a linearly ordered, open refinement $W$ of $V$ such that for each $W \in W$, the family $\left\{W^{\prime}: W^{\prime} \in W, W^{\prime}<W\right\}$ is locally finite at each point of $\bar{W}$. Since $W$ is a refinement of $V$, for each $W \in W$ there exists a $\gamma_{W} \in F$ such that $W \subseteq V_{\gamma_{W}}$. Then, $f^{-1}(W) \subseteq f^{-1}\left(V_{\gamma_{W}}\right) \subseteq \underset{\alpha \in \gamma_{W}}{U} U_{\alpha}$. Let $B=\left\{f^{-1}(W) \cap U_{\alpha}: W \in W, \alpha \in \gamma_{W}\right\}$. Obviously, $B$ is an open refinement of $u$. Order $B$ as follows: if $W \neq W^{\prime}$, then $f^{-1}(W) \cap U_{\alpha}<f^{-1}\left(W^{\prime}\right) \cap U_{\alpha^{\prime}}$ for any $\alpha \in \gamma_{W}$ and $\alpha^{\prime} \in \gamma_{W^{\prime}}$ if $W<W^{\prime}$ and $f^{-1}(W) \cap U_{\alpha}<f^{-1}(W) \cap U_{\alpha^{\prime}}$ if $\alpha<\alpha^{\prime}$ (for any $W$, $\left\{f^{-1}(W) \cap U_{\alpha}: \alpha \in \gamma_{W}\right\}$ consists of finitely many members $\}$. Now, let $f^{-1}(W) \cap U_{\alpha} \in B$ and let $x \in \overline{f^{-1}(W) \cap U_{\alpha}}$. Then

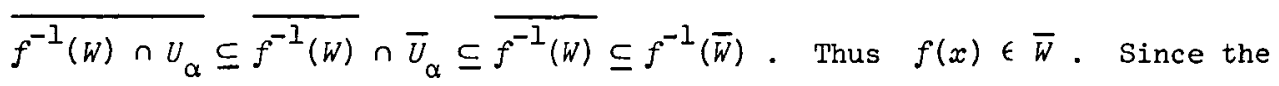
family $W^{\prime}=\left\{W^{\prime}: W^{\prime}<W\right\}$ is locally finite at each point of $\bar{W}$, therefore there exists an open set $M$ such that $f(x) \in M$ and $M$ intersects finitely many $W^{\prime}$ such that $W^{\prime}<W$. Thus $x \in f^{-1}(M)$ and $f^{-1}(M)$ can intersect at most finitely many members of $B$ which precede $f^{-1}(W) \cap U_{\alpha}$. Hence $X$ is order paracompact.

COROLLARY. (McCandless, [4]). The product of an order paracompact space with a compact space is order paracompact.

REMARK. Theorem 2 and the corollary above remain true if 'order paracompact' is replaced by 'strong order paracompact' or 'weak order paracompact'. The same is true for Theorems 4 and 5 of McCandless [4].

Motivated by the definition of order locally finite families introduced by Katuta [3], we introduce the concepts of order closure preserving families and order cushioned refinements. Throughout the sequel we shall be concerned with well ordered families.

DEFINITIONS. Let $U$ be a family of subsets of a space $X$ well ordered by $'<1 . U$ is said to be order closure preserving if for every 
$U \in U$ and every subfamily $U^{\prime}$ of $\left\{U^{\prime}: U^{\prime}<U\right\}$, we have

$$
\mathrm{Cl}_{U}\left[U\left\{U^{\prime} \cap U: U^{\prime} \in U^{\prime}\right\}\right]=U\left\{\mathrm{Cl}_{U}\left(U^{\prime} \cap U\right): U^{\prime} \in U^{\prime}\right\} .
$$

$U$ is said to be order cushioned in another family $V$ with cushion map $f: u \rightarrow U$ if for every $U \in U$ and every sub-family $u^{\prime}$ of $\left\{U^{\prime}: U^{\prime}<U\right\}$, we have

$$
\mathrm{Cl}_{U}\left[U\left\{U^{\prime} \cap U: U^{\prime} \in U^{\prime}\right\}\right] \subseteq U\left\{f\left(U^{\prime}\right): U^{\prime} \in U^{\prime}\right\} .
$$

THEOREM 3. For a regular space $X$, the following are equivalent:

(a) $X$ is paracompact;

(b) every open covering of $X$ has an order locally finite relative to a well order) open refinement;

(c) every open covering of $X$ has an order closure preserving open refinement;

(d) every open covering of $X$ has an order cushioned open refinement.

Proof. $(a) \Rightarrow(b)$ is obvious.

$(b) \Rightarrow(c)$. Let $V$ be any open covering of $X$ and let $U$ be an order locally finite (with respect to a well order) open refinement of $V$ : Now, let $U^{\prime}$ be any subfamily of $U$ such that there exists $U \in U$ such that $U^{\prime}<U$ for all $U^{\prime} \in U^{\prime}$. Then $\left\{U^{\prime} \cap U: U^{\prime} \in U^{\prime}\right\}$ is locally finite (in $U$ ) at each point of $U$. Since every locally finite family is closure preserving, we have

$$
\mathrm{Cl}_{U}\left[U\left\{U \cap U^{\prime}: U^{\prime} \in U^{\prime}\right\}\right]=U\left\{\mathrm{Cl} U_{U}\left(U^{\prime} \cap U\right): U^{\prime} \in U^{\prime}\right\} \text {. }
$$

Hence $U$ is an order colsure preserving, open refinement of $U$.

$(c) \Rightarrow(d)$. Let $V$ be any open covering of $X$. Since $X$ is regular, there exists another open covering $W$ of $V$ such that the family of closures of members of $W$ forms a refinement of $V$. Then if $U$ is an order closure preserving open refinement of $W$, then $U$ is order cushioned in $V$.

$(d) \Rightarrow(a)$. Let $U$ be any open covering of $X$. Let $V$ be an order cushioned, open refinement of $u$ with cushion map $f: v \rightarrow U$. For each 
$V \in V$, let $W_{V}=V \backsim U\left\{V^{\prime}: V^{\prime}<V\right\}$. Let $W=\left\{W_{V}: V \in V\right\}$. We shall show that $W$ is a cushioned refinement of $U$ with cushion map $g: w \rightarrow U$ defined as $g\left(W_{V}\right)=f(V)$ for each $W_{V} \in W$. If $x \in X$ and if $V$ is the smallest member of $V$ containing $x$, then $x \in W_{V}$. Let $W^{\prime}$ be any subfamily of $W$. It will be proved that $\bar{U}\left\{W_{V}: W_{V} \in W^{\prime}\right\} \subseteq U\left\{g\left(W_{V}\right): W_{V} \in W^{\prime}\right\}$. Let $y \in \overline{U\left\{W_{V}: W_{V} \in W^{\prime}\right\}}$. There exists $V \in V$ such that $y \in V$. Let $G$ be any other open set containing $y$. Then $G \cap V$ is an open set containing $y$. Since $V \cap W_{V^{\prime}}=\varphi$ for all $V^{\prime}>V$, therefore $G \cap V$ must have non-empty intersection with $U\left\{W_{V}: V^{\prime}<V, W_{V^{\prime}} \in W^{\prime}\right\}$. Therefore if $W^{\prime \prime}=\left\{W_{V}: V^{\prime}<V, W_{V^{\prime}} \in W^{\prime}\right\}$, then $y \in \overline{U\left\{W_{V}: W_{V^{\prime}} \in W^{\prime \prime}\right\}}$. Let $V^{\prime \prime}=\left\{V^{\prime} \in V: W_{V^{\prime}} \in W^{\prime \prime}\right\}$. For all $V^{\prime} \in V^{\prime \prime}, V^{\prime}<V$. Hence $\mathrm{Cl}_{V}\left[\left(U\left\{V^{\prime}: V^{\prime}<V, V^{\prime} \in V^{\prime \prime}\right\}\right) \cap V\right]=\mathrm{Cl}_{V}\left[U\left\{V^{\prime} \cap V: V^{\prime} \in V^{\prime \prime}\right\}\right]$ $\subseteq U\left\{f\left(V^{\prime}\right): V^{\prime} \in V^{\prime \prime}\right\}=U\left\{g\left(W_{V^{\prime}}\right): W_{V^{\prime}} \in W^{\prime \prime}\right\} \subseteq U\left\{g\left(W_{V^{\prime}}\right): W_{V^{\prime}} \in W^{\prime}\right\}$.

Since $V$ is an open set, therefore by Lemma 1 of McCandless [4], we have, $\mathrm{Cl}_{V}\left[\left(U\left\{V^{\prime}: V^{\prime} \in V^{\prime \prime}\right\}\right) \cap V\right]=\overline{U\left\{V^{\prime}: V^{\prime} \in V^{\prime \prime}\right\}} \cap V$.

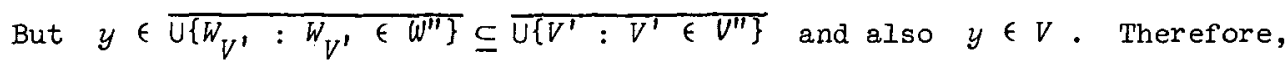
$y \in C I_{V}\left[\left(U\left\{V^{\prime}: V^{\prime} \in U^{\prime \prime}\right\}\right) \cap V\right] \subseteq U\left\{g\left(W_{V^{\prime}}\right): W_{V^{\prime}} \in W^{\prime}\right\}$. Hence

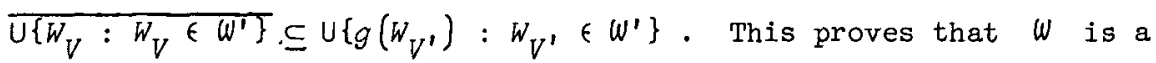
cushioned refinement of $U$ and hence $X$ is paracompact in view of Michael [6, Theorem 1.1].

This completes the proof of the theorem.

\section{References}

[1] R.H. Bing, "Metrization of topological spaces", Canad. J. Math. 3 (1951), 175-186. 
[2] Ben Fitzpatrick, Jr and Ralph M. Ford, "On the equivalence of small and large inductive dimension in certain metric spaces", Duke Math. J. 34 (1967), 33-37.

[3] Yûkiti Katuta, "A theorem on paracompactness of product spaces", Proc. Japan Acad. 43 (1967), 615-618.

[4] Bryon H. McCandless, "On order paracompact spaces", Canad. J. Math. 21 (1969), 400-405.

[5] Ernest Michael, "A note on paracompact spaces", Proc. Amer. Math. Soc. 4 (1953), 831-838.

[6] E. Michael, "Yet another note on paracompact spaces", Proc. Amer. Math. Soc. 10 (1959), 309-314.

[7] Hisahiro Tamano, "On paracompactness", Pacific J. Math. 10 (1960), 1043-1047.

[8] Jerry E. Vaughan, "Linearly ordered collections and paracompactness", (to appear).

Institute of Advanced Studies,

Meerut University,

India,

and

Faculty of Mathematics,

University of Delhi,

India. 